\title{
Vivendo com a Violência: Relatos de Casos contra a Mulher
}

\author{
Living with Violence: Case Reports against Women
}

Viviendo con Violencia: Informes de Casos contra Mujeres

Tânia Adas SALIBA

Departamento de Odontologia Preventiva e Restauradora, Faculdade de Odontologia, UNESP Univ. Estadual Paulista, 16015-050 Araçatuba - SP, Brasil

https://orcid.org/0000-0003-1327-2913

Cléa Adas Saliba GARBIN

Departamento de Odontologia Preventiva e Restauradora, Faculdade de Odontologia, UNESP Univ. Estadual Paulista, 16015-050 Aracatuba - SP, Brasil

https://orcid.org/0000-0001-5069-8812

Fernando Yamamoto CHIBA

Departamento de Odontologia Preventiva e Restauradora, Faculdade de Odontologia, UNESP Univ. Estadual Paulista, 16015-050 Araçatuba - SP, Brasil

https://orcid.org/0000-0003-4406-405X

Isabella Andrade DIAS

Departamento de Odontologia Preventiva e Restauradora, Faculdade de Odontologia, UNESP Univ. Estadual Paulista, 16015-050 Araçatuba - SP, Brasil

Artênio José Ísper GARBIN

Departamento de Odontologia Preventiva e Restauradora, Faculdade de Odontologia, UNESP Univ. Estadual Paulista, 16015-050 Araçatuba - SP, Brasil

https://orcid.org/0000-0002-7017-8942

\section{Resumo}

Introdução: No Brasil, presencia-se e convive-se com várias formas de violência contra a mulher, constituindo-se em um problema social refletido em todas as camadas da sociedade. Entre os diversos tipos de agressões infligidas contra a mulher, as de natureza física, que atingem a região da cabeça e pescoço, apresentam alta prevalência. Objetivo: Relatar oito casos de violência contra a mulher, registrados na Delegacia de Defesa da Mulher de um município do estado de São Paulo, em 2017. Métodos: Realizou-se um levantamento das informações referentes aos depoimentos coletados por meio de entrevista de oito vítimas da violência, cujas agressões acometeram a região da cabeça e pescoço, e que tiveram como resultantes lesões graves ou gravíssimas. Resultado: Observou-se que houve ampla variação em relação à idade das vitimadas (14 a 53 anos) e que, frequentemente, a agressão foi causada pelo parceiro/ex-parceiro íntimo/familiar, em ambiente doméstico, durante o período noturno. O consumo de álcool e drogas pode ser apontado como fator de risco para a perpetuação desse fenômeno. Como consequências físicas das agressões, foram relatadas lesões corporais na região de cabeça e pescoço, com lacerações, escoriações, contusões, luxações, e hematomas envolvendo a face, ouvidos, olhos, lábios e cavidade oral, além da perda de elementos dentários. Conclusão: Confirma-se a importância do registro da ocorrência pela vítima da agressão, pois esta documentação subsidiará o acolhimento da vítima, encaminhamento para tratamento específico, e abertura de processo contra o agressor. Destaca-se a importância das instituições públicas na prevenção e intervenção da violência contra a mulher.

Descritores: Violência Contra a Mulher; Relato de Casos; Ferimentos e Lesões.

\section{Abstract}

Introduction: In Brazil, various forms of violence against women are witnessed and experienced, constituting a social problem reflected in all layers of society. Among the types of aggressions inflicted on women, those of a physical nature, which affect the head and neck region, are highly prevalent. Objective: To report eight cases of violence against women, registered at the Women's Defense Police Station in a municipality in the state of São Paulo, in 2017. Methods: A survey was made of the information regarding the testimonies collected through interviews with eight victims of violence, whose aggressions affected the head and neck region, and which resulted in serious or very serious injuries. Result: It was observed that there was wide variation in the age of the victims (14 to 53 years old) and that, frequently, the aggression was caused by the partner/ex-intimate partner/family member, in domestic environment, at night. The consumption of alcohol and drugs can be identified as risk factors for the perpetuation of this phenomenon. As physical consequences of the aggressions, bodily injuries were reported in the head and neck region, with lacerations, abrasions, dislocations, and bruises involving the face, ears, eyes, lips and oral cavity, in addition to the tooth loss. Conclusion: The importance of registering the occurrence by the victim of the aggression is confirmed, as this documentation will subsidize the reception, referral for specific treatment, and opening of proceedings against the aggressor. The importance of public institutions in the prevention and intervention of violence against women is highlighted.

Descriptors: Violence Against Women; Case Reports; Wounds and Injuries.

\section{Resumen}

Introducción: En Brasil, presenciamos y vivimos diversas formas de violencia contra la mujer, constituyendo un problema social reflejado en todos los estratos de la sociedad. Entre los diversos tipos de agresiones infligidas, las de carácter físico, que afectan región de cabeza y cuello, tienen alta prevalencia. Objetivo: Reportar ocho casos de violencia contra la mujer, registrados en la Comisaría de Defensa de la Mujer de un municipio del estado de São Paulo, en 2017. Métodos: Se realizó un relevamiento de información sobre testimonios recolectados al entrevistar ocho víctimas de violencia, cuyas agresiones afectaron la región de cabeza y cuello, que resultaron en lesiones graves o muy graves. Resultado: Se observó que hubo amplia variación en relación a edad de las víctimas (14 a 53 años) y que, frecuentemente, la agresión fue provocada por la pareja/expareja íntima/ familiar, en ámbito doméstico, durante la noche. El consumo de alcohol y drogas se puede identificar como factores de riesgo para la perpetuación de este fenómeno. Como consecuencia física de las agresiones se reportaron lesiones corporales en región de cabeza y cuello: laceraciones, abrasiones, contusiones, dislocaciones y contusiones en rostro, orejas, ojos, labios y cavidad bucal, además pérdida de elementos dentarios. Conclusión: Se confirma la importancia de registrar la ocurrencia de la agresión, ya que esta documentación subsidiará la recepción de la víctima, derivación para tratamiento específico y la apertura de procesos contra el agresor. Se destaca la importancia de las instituciones públicas en la prevención e intervención de la violencia contra la mujer.

Descriptores: Violencia Contra la Mujer; Informes de Casos; Heridas y Traumatismos.

INTRODUÇÃO

A violência contra as mulheres é uma das principais violações dos direitos humanos e evidencia um grave problema social, cultural e de saúde pública, com efeitos significativos em curto, médio e longo prazo que afetam a saúde física, mental e o bem-estar das mulheres, crianças e famílias ${ }^{1,2}$. De acordo com a Organização Mundial da Saúde, globalmente, estima-se que $27 \%$ das mulheres entre 15 e 49 anos, já sofreram violência física e/ou sexual alguma vez na vida, indicando que, aproximadamente, 753 milhões de mulheres foram submetidas violência física e/ou sexual pelo menos uma vez desde os 15 anos $^{3}$. No Brasil, esses dados também são alarmantes, estimando-se que $23 \%$, ou seja, mais de uma a 
cada cinco mulheres brasileiras, já sofreram violência física e/ou sexual alguma vez na vida ${ }^{3}$.

Desse modo, constata-se que a violência contra a mulher é um problema de saúde pública de proporções epidêmicas no Brasil e, portanto, não pode ser tratado como se fosse restrito a alguns segmentos, uma vez que permeia toda a sociedade ${ }^{4}$. A prevenção e 0 enfrentamento da violência contra a mulher passam, necessariamente, pela redução das desigualdades de gênero $e$ requerem 0 engajamento de diferentes setores da sociedade para garantir que todas as mulheres tenham acesso ao direito básico de viver sem violência ${ }^{5}$.

Visando prevenir, enfrentar e erradicar a violência contra a mulher, foram criadas, a partir de 1985, as Delegacias Especializadas de Atendimento à Mulher (DEAMs), para servirem como referência na busca de apoio e segurança para as mulheres, atuando de forma integrada com as Secretarias Estaduais de Segurança Pública e oferecendo atendimento qualificado, evitando a revitimização da mulher ${ }^{6}$. Os serviços de saúde também têm um papel fundamental na resposta à violência contra as mulheres, pois muitas vezes constitui-se no primeiro local onde as vítimas buscam atendimento. Assim, é importante que estes serviços estejam disponíveis ininterruptamente e que os profissionais dos de saúde estejam capacitados para realizar 0 acolhimento adequado das vítimas e a notificação dos casos de violência ${ }^{7}$.

O objetivo neste estudo foi relatar oito casos de violência contra as mulheres, atingidas na região da cabeça e pescoço, com resultantes graves ou gravíssimas, registrados na Delegacia de Defesa da Mulher de um município do estado de São Paulo, em 2017.

MATERIAL E MÉTODO

Trata-se de um estudo de relato de caso de mulheres vítimas de violência, cujas agressões tiveram acometimento da região da cabeça e pescoço, com consequências graves ou gravíssimas. A pesquisa foi realizada com mulheres que registraram a ocorrência em uma Delegacia de Defesa da Mulher do município de Bauru-SP, no período de março a julho de 2017. As mulheres que concordaram em participar da pesquisa tiveram seus depoimentos coletados por uma única pesquisadora previamente treinada, por meio de entrevistas individuais realizadas em uma sala reservada da Delegacia, imediatamente após o registro do boletim de ocorrência. A entrevista foi organizada visando a obtenção de informações sobre as seguintes variáveis: autor da agressão; forma de agressão; local da ocorrência; natureza da lesão; região do corpo acometida; horário e dia da semana da ocorrência; escolaridade da vítima; ocupação da vítima; ingestão de bebida alcoólica pela vítima e/ou pelo agressor; atendimento anterior da mesma ocorrência em unidade de saúde.

De acordo com o Código Penal Brasileiro, em seu artigo 129, as lesões corporais são classificadas quanto a natureza como leve, grave ou gravíssima ${ }^{8}$. A lesão corporal de natureza leve é aquela que não causa grande ofensa à integridade corporal, embora, deixe um trauma psicológico muito grande. É considera lesão corporal de natureza grave, a agressão que resulta em incapacidade para as ocupações habituais por mais de trinta dias; perigo de vida; debilidade permanente de membro, sentido ou função; e aceleração de parto. As lesões gravíssimas são aquelas em que as resultantes demonstram a incapacidade permanente para o trabalho; enfermidade incurável; perda ou inutilização de membro, sentido ou função; deformidade permanente; e aborto.

O presente estudo foi aprovado pelo Comitê de Ética da Faculdade de Odontologia de Araçatuba-UNESP, processo 2003/535 e foi conduzido seguindo todas as normas estabelecidas na resolução no $466 / 2012$ do Conselho Nacional de Saúde.

RESULTADOS E DISCUSSÃO

Os relatos analisados foram fornecidos espontaneamente pelas mulheres e, como na maioria dos casos, as vítimas são encaminhadas para o atendimento psicológico, poucas concordaram em participar da entrevista, pois teriam que relatar 0 caso ocorrido mais uma vez. As vítimas que concordaram em participar da pesquisa demonstraram estar muito nervosas, entretanto, aos poucos, com o decorrer da entrevista pôdese perceber nas vítimas uma grande vontade de falar e de "desabafar" sobre suas revoltas, dores e condições de vida.

Os efeitos da violência contra as mulheres se fazem sentir, principalmente, em indivíduos mais vulneráveis, incluindo grupos populacionais que apresentam condições de baixo nível de escolaridade e menor condição socioeconômica, entretanto, isso não significa que os estratos sociais mais privilegiados não sejam afetados ${ }^{9,10}$. A amplitude do problema pôde ser percebida pelas variadas características socioeconômicas das vítimas, que envolviam mulheres com diferentes níveis 
de escolaridade e ocupação, incluindo desde pessoas que nunca haviam frequentado a escola até as que possuíam ensino superior completo.

A partir dos relatos das vítimas foi possível compreender a extensão e as consequências das agressões envolvendo a região de cabeça e pescoço. A face caracterizase por ser uma região de grande complexidade anatômica e funcional e que, frequentemente, está relacionada a traumatismos decorrentes de agressões $^{11}$. Esta vulnerabilidade evidencia que a estrutura facial requer atenção especial por parte dos profissionais de saúde envolvidos no atendimento de mulheres vítimas de violência, considerando inclusive a sua importância no convívio social e nas relações interpessoais ${ }^{12}$. Os achados deste estudo estão de acordo com os resultados de outras pesquisas sobre violência contra mulheres, que demonstraram alta prevalência de lesões na região de cabeça e pescoço, e que as áreas da face acometidas com maior frequência são a região periorbitária, região frontal e os dentes ${ }^{13,14}$. A realização contínua e sistemática de estudos sobre a prevalência, severidade e fatores relacionados às lesões da região da cabeça e pescoço pode ter um papel importante no enfrentamento da violência contra as mulheres, pois a partir das análises dessas ocorrências é possível nortear a elaboração de políticas de promoção e prevenção da saúde, além de avaliar o progresso, o impacto e a eficácia das estratégias implementadas.

Ao analisar os dados coletados nas entrevistas relativos à idade das vítimas, constatou-se que não existem parâmetros para que esse tipo de agressão ocorra, sugerindo que qualquer mulher, em qualquer etapa do ciclo de vida, está sujeita a essas ações. No presente estudo, notou-se que, em grande parte dos relatos, o agressor era o parceiro íntimo atual/passado ou um familiar da vítima, e que a agressão ocorreu em sua própria residência. A violência doméstica contra a mulher é uma preocupação crescente na sociedade mundial, pois esta forma de violência, que ocorre no núcleo familiar das vítimas e no âmbito do próprio lar configura-se em um fenômeno complexo, envolvendo uma gama de fatores que contribuem para a sua perpetuação, tais como dependência financeira, medo, filhos, insegurança e impunidade, de modo que muitas vítimas precisam de ajuda para se libertar dessa condição $^{15-17}$. Nesse sentido, salienta-se a necessidade de ações efetivas no enfrentamento da violência contra as mulheres, não apenas em relação à rígida aplicação de punição aos agressores, como também para o apoio e recuperação da dignidade $e$ continuidade da vida das vítimas.

A partir do relato das vítimas, foram identificados fatores relevantes que podem contribuir para o planejamento de políticas públicas voltadas para 0 enfrentamento da violência contra a mulher: a) a ocorrência dos casos durante os finais de semana, bem como nos períodos da noite e madrugada, reforçando a importância de que os serviços de segurança e saúde voltados ao atendimento das mulheres vítimas de violência funcionem em horários diferenciados, cobrindo ininterruptamente os períodos de maior demanda; b) algumas vítimas não recorreram imediatamente à Delegacia da mulher para registrar a ocorrência pois, em decorrência da gravidade da agressão, procuraram primeiramente uma unidade de saúde ou hospital, evidenciando a importância da capacitação dos profissionais e serviços de saúde para 0 atendimento e notificação dos casos de violência, possibilitando o acesso adequado e oportuno da vítima aos serviços de segurança pública e justiça para a obtenção de medidas protetivas ${ }^{5,18,19}$.

Outros fatores agravantes associados às ocorrências de violência registradas nesse estudo foram o consumo de drogas ilícitas e a ingestão de bebidas alcoólicas. Este achado é corroborado por outras pesquisas que indicam que o uso abusivo de álcool e drogas pelo companheiro íntimo ou familiar pode potencializar a violência contra a mulher e salientam a importância da articulação das medidas de atenção à mulher em situação de violência doméstica com ações de prevenção de consumo de álcool e outras drogas por seus acompanhantes ${ }^{20-22}$

A violência presente nas relações de gênero é um sério problema de saúde pública para mulheres de todo o mundo e as agressões, sejam elas físicas, verbais, morais ou sexuais, podem resultar em profundas cicatrizes físicas e psicológicas. Evidências sugerem que mulheres vítimas de violência têm sua saúde prejudicada tanto pelas lesões e sequelas físicas resultantes da agressão quanto por desenvolverem estresse, ansiedade, depressão e baixa autoestima $^{13,23,24}$.

A elaboração das ações para prevenção da violência contra a mulher deve ser norteada por evidências que potencializem os recursos aplicados para a execução das estratégias adotadas, ressaltando a importância da realização contínua de estudos que identifiquem os fatores associados e as consequências da violência contra a mulher. 


\section{CONCLUSÃO}

Conclui-se que a violência contra a mulher é um problema complexo e multifatorial, que pode afetar todas as camadas da sociedade, de modo que as políticas públicas devem incluir a atuação articulada de diversas áreas, tais como direitos humanos, educação, saúde, justiça e segurança pública. Os serviços de saúde têm um papel de grande relevância, pois muitas vezes representam o primeiro contato da vítima com as instituições públicas e, portanto, devem estar capacitados para realizar o acolhimento e a notificação do caso, contribuindo para que a vítima encontre 0 caminho da superação da condição em que se encontra.

\section{REFERÊNCIAS}

1. Souza TMC, Rezende FF. Violência contra mulher: concepções e práticas de profissionais de serviços públicos. Est Inter Psicol. 2018;9:21-38.

2. Bott S, Guedes A, Ruiz-Celis AP, Mendoza JA. Intimate partner violence in the Americas: a systematic review and reanalysis of national prevalence estimates. Rev Panam Salud Publica. 2019;43:e26.

3. World Health Organization. Violence against women prevalence estimates, 2018. Global, regional and national prevalence estimates for intimate partner violence against women and global and regional prevalence estimates for non-partner sexual violence against women. Geneva: World Health Organization, 2021. https://cdn.who.int/media/docs/defaultsource/documents/violenceprevention/vaw_report_web_09032021_oleksa ndr.pdf?sfvrsn=a82ef89c_5\&download=true

4. Garbin CAS, Saliba TA, Chiba FY, Garbin AJI. Violência dimensionada: análise da notificação. Rev Enferm UFPI. 2020;9(1).

5. Garbin CAS, Dias IA, Rovida TAS, Garbin AJI. Desafıos do profissional de saúde na notifıcação da violência: obrigatoriedade, efetivação e encaminhamento. Ciênc Saúde Coletiva. 2015;20(6):1879-90.

6. Souza TMC, Martins TF. Vivências de policiais de uma DEAM no Sudoeste Goiano. Fractal Rev Psicol. 2021;33:21-30.

7. Garbin CAS, Rovida TAS, Costa AA, Garbin AJl. Percepção e atitude do cirurgião-dentista servidor público frente à violência intrafamiliar em 24 municípios do interior do estado São Paulo, 2013-2014. Epidemiol Serv Saúde. 2016; 25(1):179-86.

8. Brasil. Código Penal. Decreto-Lei no 2.848, de 7 de dezembro de 1940. http://www.planalto.gov.br/ccivil_03/decretolei/del2848compilado.htm
9. Haobijam S, Singh KA. Socioeconomic determinants of domestic violence in northeast India: Evidence from the National Family Health Survey (NFHS-4). J Interpers Violence. 2021: 8862605211005133.

10. Pereira MUL, Gaspar RS. Socioeconomic factors associated with reports of domestic violence in large brazilian cities. Front Public Health. 2021;9:623185.

11. Siber S, Matijević M, Sikora M, Leović D, Mumlek I, Macan D. Assessment of oromaxillofacial trauma according to gender, age, cause and type of the injury. Acta Stomatol Croat. 2015; 49(4): 340-47.

12. Braun TL, Maricevich RS. Soft tissue management in facial trauma. Semin Plast Surg. 2017;31(2): 73-9.

13. Garbin CAS, Garbin AJI, Dossi AP, Dossi MO. Violência doméstica: análise das lesões em mulheres. Cad Saúde Pública. 2006;22(12):2567-73.

14. Thomas R, Dyer GSM, Tornetta lii P, Park $H$, Gujrathi R, Gosangi B, Lebovic J, Hassan N, Seltzer SE, Rexrode KM, Boland GW, Harris $\mathrm{MB}$, Khurana B. Upper extremity injuries in the victims of intimate partner violence. Eur Radiol. 2021:1-8.

15. Souza MB, Silva MFS. Estratégias de enfrentamento de mulheres vítimas de violência doméstica: uma revisão da literatura brasileira. Pensando Fam. 2019;23(1),153-66.

16. Silva EF, Santos SEB, Gomes WS, Santana AM. Violência contra a mulher: ação clínica na prevenção em saúde. Saúde Desenvolv Hum. 2019;7(2):77-87.

17. Villela WV, Vianna LAC, Lima LFP, Sala DCP, Vieira TF, Vieira ML, Oliveira EM. Ambiguidades e contradições no atendimento de mulheres que sofrem violência. Saude Soc. $2011 ; 20(1): 113-23$.

18. Guimarães MC, Pedroza RLS. Violência contra a mulher: problematizando definições teóricas, filosóficas e jurídicas. Psicol Soc. 2015;27:256-66.

19. Garcia LP, Duarte EC, Freitas LRS, Silva GDM. Violência doméstica e familiar contra a mulher: estudo de casos e controles com vítimas atendidas em serviços de urgência e emergência. Cad Saúde Pública. 2016;32:e00011415.

20. Krishnakumar A, Verma S. Understanding domestic violence in India During COVID-19: a routine activity approach. Asian J Criminol. 2021:1-17.

21. Siria S, Leza L, Fernández-Montalvo J, Echauri JA, Azkarate JM, Martínez M. Differential psychopathological profile of male intimate partner violence perpetrators depending on Problematic alcohol use. Addict Behav. 2021;118:106887. 
22. Vieira LB, Cortes LF, Padoin SM, Souza IE, de Paula CC, Terra MG. Abuso de álcool e drogas e violência contra as mulheres: denúncias de vividos. Rev Bras Enferm. 2014;67(3):366-72.

23. Al Kendi A, Al Shidhani N, Al Kiyumi M. Domestic violence among Omani women: prevalence, risk factors and help-seeking behaviour. East Mediterr Health J. 2021;27(3):242-49.

24. Hameed $M$, O'Doherty $L$, Gilchrist $G$, Tirado-Muñoz J, Taft A, Chondros P, Feder G, Tan M, Hegarty K. Psychological therapies for women who experience intimate partner violence. Cochrane Database Syst Rev. 2020;7(7):CD013017.

\section{CONFLITO DE INTERESSES}

Os autores declaram não haver conflitos de interesse

\section{AUTOR PARA CORRESPONDÊNCIA}

\section{Fernando Yamamoto Chiba}

Departamento de Odontologia Preventiva e Restauradora, Faculdade de Odontologia, UNESP Univ. Estadual Paulista, 16015-050

Araçatuba - SP, Brasil

e-mail: fernando.chiba@unesp.br 